\title{
Inflamação idiopática da órbita com extensão extra-orbital: relato de caso
}

\section{Idiopathicorbital inflammation with extraorbital extension:case report}

\author{
Tânia Pereira Nunes ${ }^{1}$ \\ Ricardo Roizemblatt ${ }^{2}$ \\ Gustavo Miki ${ }^{3}$ \\ Renato Garcia ${ }^{4}$ \\ Ruth Miyuki Santo 5 \\ Edilberto Olivalves ${ }^{6}$ \\ Suzana Matayoshi ${ }^{7}$
}

\section{RESUMO}

Relato de caso de uma paciente com inflamação idiopática da órbita com extensão extra-orbital. Foi realizada biópsia para confirmar o diagnóstico e a tomografia computadorizada demonstrou o comprometimento extraorbital do processo inflamatório. O tratamento foi feito com metotrexato e radioterapia.

Descritores: Granuloma de células plasmáticas orbital; Neoplasias orbitárias/quimioterapia; Radioterapia adjuvante; Metotrexato; Relatos de casos [tipo de publicação]

\section{INTRODUÇÃO}

Inflamação idiopática da órbita (IIO), também chamada de pseudotumor, representa um processo inflamatório não granulomatoso que pode acometer qualquer tecido orbital, sem causa local ou sistêmica definida. É a terceira doença orbital mais comum, depois de orbitopatia distireoidiana e doença linfoproliferativa ${ }^{(1)}$. Seu diagnóstico é feito por exclusão baseado na história, exame clínico, resultados de exames complementares que possam diagnosticar outra doença, por exemplo, testes laboratoriais (T3, T4, TSH, VHS, glicemia, uréia, creatinina, hemoculturas), tomografia computadorizada, resposta à medicação e biópsia ${ }^{(1)}$.

A biópsia deve ser indicada em casos que o diagnóstico é duvidoso baseando-se apenas no quadro clínico e nas imagens, quando há acometimento da porção anterior da órbita, pela facilidade de acesso, nos casos em que há recorrência ou resistência ao tratamento ${ }^{(1-2)}$.

A doença pode apresentar-se de forma aguda, subaguda ou crônica, sendo que as manifestações inflamatórias são menos expressivas ou ausentes nas duas últimas ${ }^{(2)}$. É mais freqüente em adultos e pode acometer ambos os $\operatorname{sexos}^{(1)}$. A apresentação unilateral é mais comum, mas pode ser bilateral, principalmente em criança.

A IIO pode ser classificada com base no tecido orbital primário envolvido: miosite (músculo extra-ocular), dacrioadenite (glândula lacrimal), perisclerite, perineurite; outra classificação baseia-se na localização: anterior (olho e órbita), apical (ápice orbital e seio cavernoso), processos inflamatórios orbitários nodulares e difuso ${ }^{(2-3)}$.

O principal diagnóstico diferencial deve ser feito com oftalmopatia de Graves. Ambas podem apresentar sinais congestivo-edematosos pálpebroconjuntivais, exoftalmia, anomalias oculomotoras e redução da acuidade visual; a oftalmopatia de Graves é bilateral com muito mais frequiência. Outras doenças que devem ser descartadas são: celulites orbitais, granulomatose de Wegener, fístula carótido-cavernosa, corpos estranhos, fungos, sarcoidose, sinusopatias, mucoceles, afecções do seio cavernoso, etc. As formas difusas e nodulares confundem-se com maior freqüência com linfo- 
mas, hemangiomas, tumores de glândula lacrimal ou outros tumores orbitais mais $\operatorname{raros}^{(1-4)}$.

O tratamento de escolha para IIO é o corticóide, com rápida melhora do quadro inflamatório. Radioterapia pode ser indicada para pacientes que não responderam ao corticóide e nos casos de evolução insidiosa ${ }^{(4)}$.

O objetivo deste estudo é relatar um caso de inflamação idiopática da órbita com extensão extra-orbital de difícil controle.

\section{RELATO DE CASO}

MM, 45 anos, feminina, foi encaminhada para o setor de Plástica Ocular do Hospital das Clínicas da Faculdade de Medicina de São Paulo, Universidade de São Paulo, com quadro de blefaroptose adquirida à direita.

Como antecedentes oftalmológicos, havia um tratamento realizado para esclerite bilateral entre 1993 e 1995, evoluindo com atrofia bulbar à direita. Em 1995, foi submetida à cirurgia de catarata com implante de lente intra-ocular à esquerda. Usuária de prótese ocular havia um ano.

No exame oftalmológico, a paciente apresentava ptose palpebral grave à direita. Era usuária de prótese escleral à direita. As medidas da rima palpebral eram: $3 \mathrm{~mm}$ à direita e $10 \mathrm{~mm}$ à esquerda. A função do músculo levantador da pálpebra superior (MLPS) era: $10 \mathrm{~mm}$ à direita e $18 \mathrm{~mm}$ à esquerda. O sulco palpebral à direita era alto (Figura 1).

A cirurgia proposta foi a reinserção da aponeurose do MLPS, pois a função do MLPS à direita era boa.

Antes da data programada para a correção da ptose pálpebral, a paciente retornou ao ambulatório com quadro de dor aguda, de forte intensidade, na órbita direita. Ao exame oftalmológico, a paciente apresentava edema palpebral à direita, quemose e congestão vascular conjuntival difusa. Foi realizada tomografia computadorizada (TC) que mostrou infiltração difusa da órbita direita. Como a investigação sistêmica foi negativa para processo infeccioso ou auto-imune, foi feito o diagnóstico de IIO direita e iniciou-se tratamento com prednisolona, $80 \mathrm{mg}$ por dia.

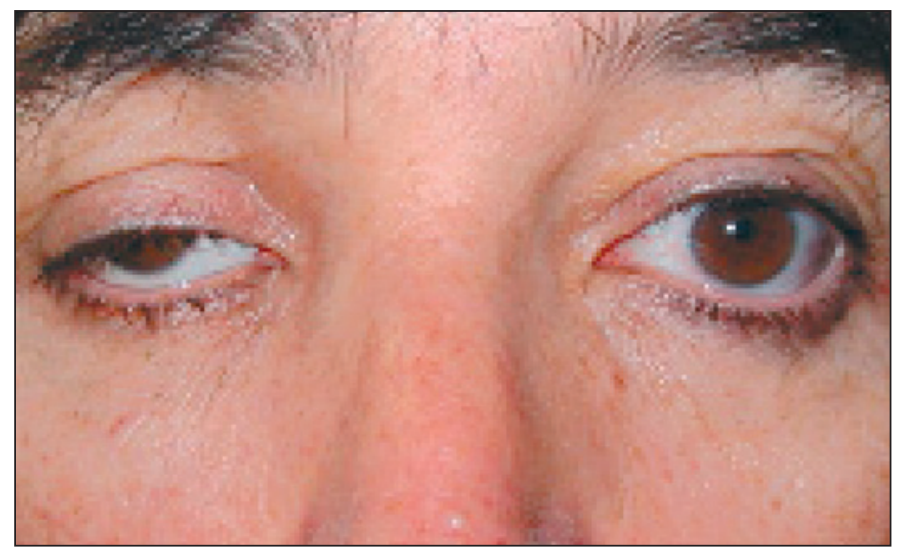

Figura 1 - Blefaroptose à direita
Nova avaliação foi feita em 4 semanas: o quadro doloroso e inflamatório orbital progrediu mesmo com a medicação em uso; optou-se assim pela realização de biópsia da órbita direita, que mostrou infiltrado inflamatório polimórfico com presença de linfócitos $\mathrm{T}$ e B, plasmócitos, histiócitos e eosinófilos confirmando o diagnóstico de IIO. Nova TC foi feita mostrando progressão do quadro infiltrativo com comprometimento das fissuras orbitais, seio cavernoso, fossas anterior e média do crânio e fossa pterigomaxilar direita (Figura 2). Haviam também erosões focais das paredes e rebordos orbitais.

Com a piora da dor e o resultado da segunda TC, optou-se pelo uso de metotrexato, com dose inicial de 7,5 $\mathrm{mg}$ por semana; nova biópsia orbital foi realizada, confirmando mais uma vez IIO, tipo esclerosante (Figura 3).

Mesmo com o aumento da dose de metotrexato para $20 \mathrm{mg}$ por semana, o quadro doloroso e inflamatório não diminuiu, optou-se pela radioterapia. Foram realizadas 10 sessões de radioterapia, dose total de $2500 \mathrm{cGy}$, no período de 10 dias, com remissão da dor e da inflamação orbital após 50 dias do tratamento.

Após 6 meses de seguimento, nova TC foi realizada mostrando estabilização do processo infiltrativo orbital. Com o controle da IIO, nova propedêutica palpebral foi realizada com o objetivo de programar a correção da blefaroptose à direita. A função do MLPS à direita era ausente, isto poderia ser explicado pelo comprometimento do nervo oculomotor pelo processo inflamatório ou pela infiltração do MLPS, e a paciente foi submetida à correção da ptose palpebral pela técnica da suspensão frontal com boa evolução (Figura 4).

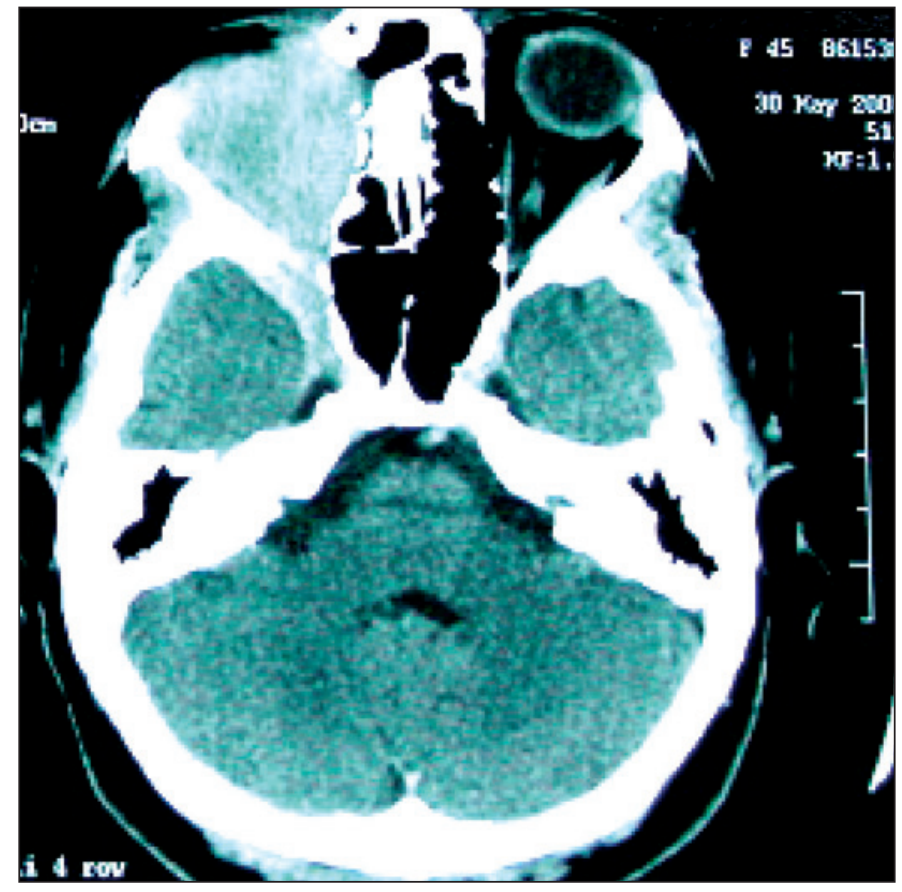

Figura 2 - Tomografia computadoriza de órbitas em corte axial mostrando processo infiltrativo difuso orbital direito com extensão para seio cavernoso 


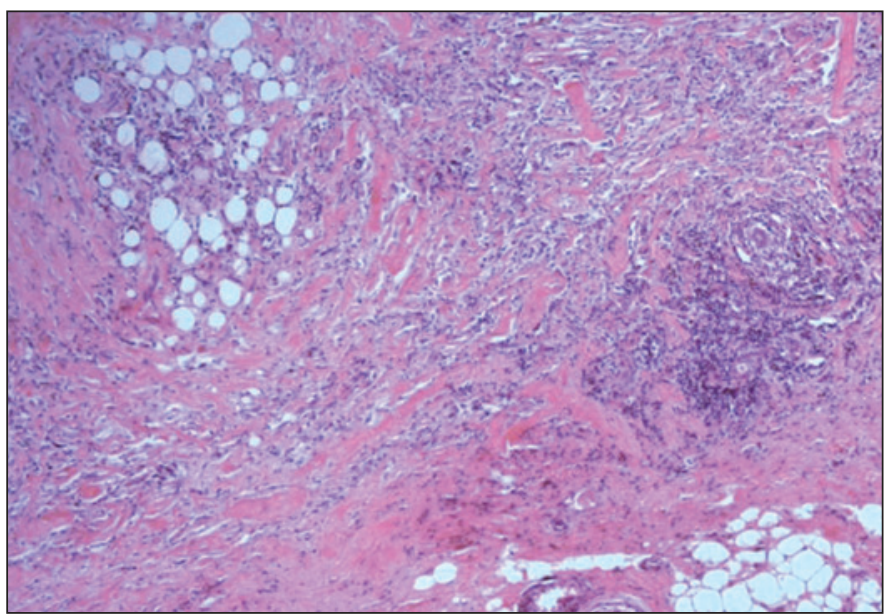

Figura 3 - Histopatológico da biópsia da órbita direita mostrando infiltrado inflamatório polimórfico, com quantidade de tecido conectivo intersticial (fibrose) em grande quantidade

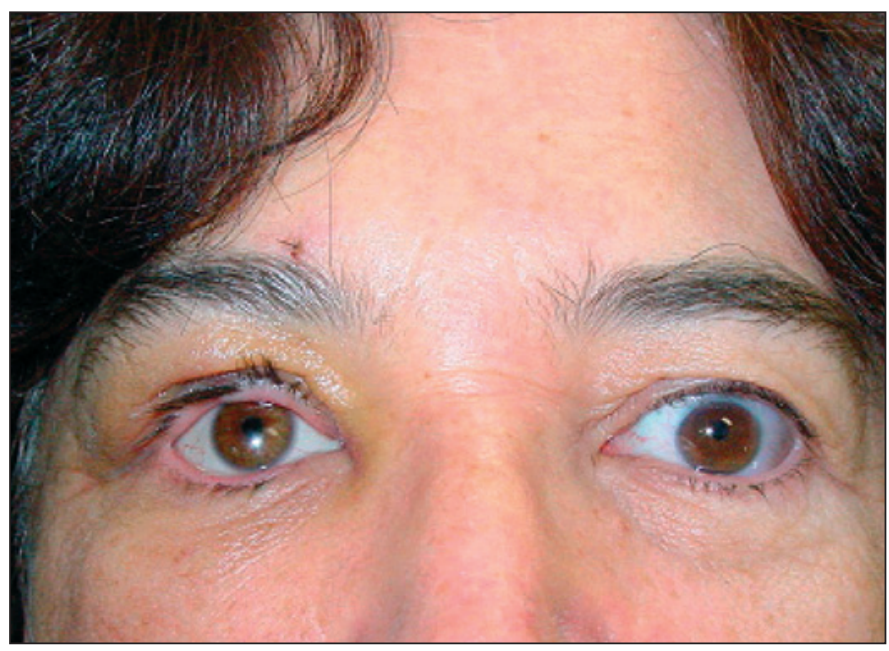

Figura 4 - Suspensão frontal: pós-operatório imediato

\section{DISCUSSÃO}

IIO com extensão extra-orbital é bastante incomum ${ }^{(2,5)}$. No presente estudo, o processo inflamatório inicialmente estava restrito à órbita e com a evolução do quadro, progrediu para seio cavernoso, fossa anterior e média do crânio e fossa pterigomaxilar. Alguns autores descreveram 2 casos de IIO tipo esclerosante com envolvimento da fossa infratemporal e pterigopalatina; um dos pacientes apresentou erosão óssea como o caso descrito neste estudo ${ }^{(5)}$. A erosão óssea pode sugerir um processo tumoral maligno, dificultando assim o diagnóstico diferencial. Com o resultado da segunda TC mostrando infiltração difusa da órbita e extensão extra-orbital, realizamos nova biópsia, pois suspeitávamos de um processo neoplásico. Em outro estudo, dor orbital não estava presente em todos os casos e a realização dos exames de imagem, como a TC, foi fundamental para a determinação da extensão extra-orbital ${ }^{(6)}$.
Outros autores descreveram a associação da miosite idiopática, que é um subtipo da IIO, com a esclerite. Todos os pacientes com miosite apresentavam esclerite posterior. Os pacientes que apresentavam esclerite e miosite tinham mais problemas oculares que aqueles que tinham somente esclerite, indicando um comprometimento e uma resposta inflamatória maior $^{(7)}$. Nossa paciente tinha uma história de esclerite bilateral de difícil controle, que evoluiu com atrofia bulbar à direita. Haveria relação entre o quadro atual de IIO e o antecedente de esclerite? Na IIO, as recidivas e remissões dos surtos inflamatórios são comuns na mesma órbita ou na órbita contralateral ao longo do tempo, especialmente nas formas miosíticas ${ }^{(2)}$.

Alguns autores acreditam que o processo de IIO do tipo esclerosante seria uma evolução do processo agudo da órbita, como neste caso relatado. Outros autores consideram o tipo esclerosante como outra entidade ${ }^{(1-2)}$.

Em nosso relato, o tratamento com corticóide não apresentou o resultado esperado, remissão da dor e do processo inflamatório orbital. $\mathrm{O}$ controle da doença foi conseguido com a radioterapia. Alguns autores acreditam que, em casos de IIO com extensão extra-orbital, o uso de altas doses de corticóide e radioterapia deva ser utilizado antes de drogas imunosupressoras ou tratamento cirúrgico ${ }^{(5)}$. A segunda opção terapêutica utilizada pela paciente deste estudo foi o metotrexato, que é uma droga imunossupressora. A dose inicial foi de 7,5 $\mathrm{mg}$ por semana e a dose máxima foi de $20 \mathrm{mg}$. Apesar de relatos demonstrarem boa resposta com o uso de metotrexato ${ }^{(8)}$, nosso caso não apresentou melhora do quadro, sendo que só houve remissão após a radioterapia.

A radioterapia é usada em pacientes com IIO resistentes ou intolerantes ao corticóide ${ }^{(4)}$. A dose normalmente utilizada é de $20 \mathrm{~Gy}$, divididas em 10 sessões durante 2 semanas $^{(1,9)}$. Com esta baixa dose, a única estrutura ocular que pode ser afetada, após a radioterapia, é o cristalino. Outras complicações como olho seco, retinopatia e neuropatia óptica são observadas com doses acima de $30 \mathrm{~Gy}^{(1)}$.

Outro ponto importante deste caso é a ptose palpebral. Antes do aparecimento da IIO, a função do MLPS à direita era boa, portanto a técnica proposta para a correção da ptose palpebral foi a reinserção da aponeurose do MLPS. Com o comprometimento das estruturas orbitais e extra-orbitais pelo processo inflamatório, a função de elevação palpebral do MLPS apresentou importante diminuição, pela infiltração local do MLPS ou pelo envolvimento do nervo oculomotor, responsável pela inervação motora do MLPS, portanto a correção da blefaroptose só foi possível com a técnica da suspensão frontal ${ }^{(10)}$.

Em conclusão, através de um caso de apresentação atípica de IIO, este estudo mostra a importância do diagnóstico definitivo, baseado em exames de imagem e da biópsia, para a adoção da terapêutica adequada e resolução da doença.

\section{ABSTRACT}

The authors report a case of a patient with idiopathic orbital inflammation with extension beyond the orbit. Biopsy was 
performed to confirm the diagnosis of idiopathic orbital inflammation and computed tomography demonstrated the extraorbital extension. Treatment with methotrexate and radiotherapy was used.

Keywords: Granuloma, plasma cell, orbital; Orbital neoplasms/ drug therapy; Methotrexate; Radiotherapy adjuvant; Case reports [publication type]

\section{REFERÊNCIAS}

1. Yuen SJ, Rubin PA. Idiopathic orbital inflammation: distribution, clinical features, and treatment outcome. Arch Ophthalmol. 200;121(4):491-9. Comment in: Arch Ophthalmol. 2004;122(7):1092; author reply 1092-3.
2. Rodrigues-Alves CA, Santo RM. Pseudotumor de órbita. In: RodriguesAlves CA. Neuroftalmologia. São Paulo: Roca; 2000. p.193-211.

3. Dantas AM. Inflamações da órbita. In: Dantas AM, Monteiro ML. Doenças da órbita. Rio de Janeiro: Cultura Médica; 2002. p.153-78.

4. Jacobs D, Galetta S. Diagnosis and management of orbital pseudotumor. Curr Opin Ophthalmol. 2002;13(6):347-51.

5. Cruz AA, Akaishi PM, Chahud F, Elias JJ. Sclerosing inflammation in the orbit and in the pterygopalatine and infratemporal fossae. Ophthal Plast Reconstr Surg. 2003;19(3):201-6.

6. Mahr MA, Salomao DR, Garrity JA. Inflammatory orbital pseudotumor with extension beyond the orbit. Am J Ophthalmol. 2004;138(3):396-400.

7. Boonman ZF, De Keizer RJ, Graniewski-Wijnands HS, Watson PG. Orbital myositis in scleritis. Br J Ophthalmol. 2003;87(1):38-42.

8. Smith JR, Rosenbaum JT. A role for methotrexate in the management of noninfectious orbital inflammatory disease. Br J Ophthalmol. 2001;85(10):1220-4.

9. Chiu CS, Rubin PA. Pharmacotherapies and nonpharmacotherapies for orbital inflammatory diseases. Int Ophthalmol Clin. 2004;44(3):165-85.

10. Matayoshi S. Blefaroptose. In: Matayoshi S, Forno EA, Moura EM. Manual de cirurgia plástica Ocular. São Paulo: Roca; 2004. p.87-107.

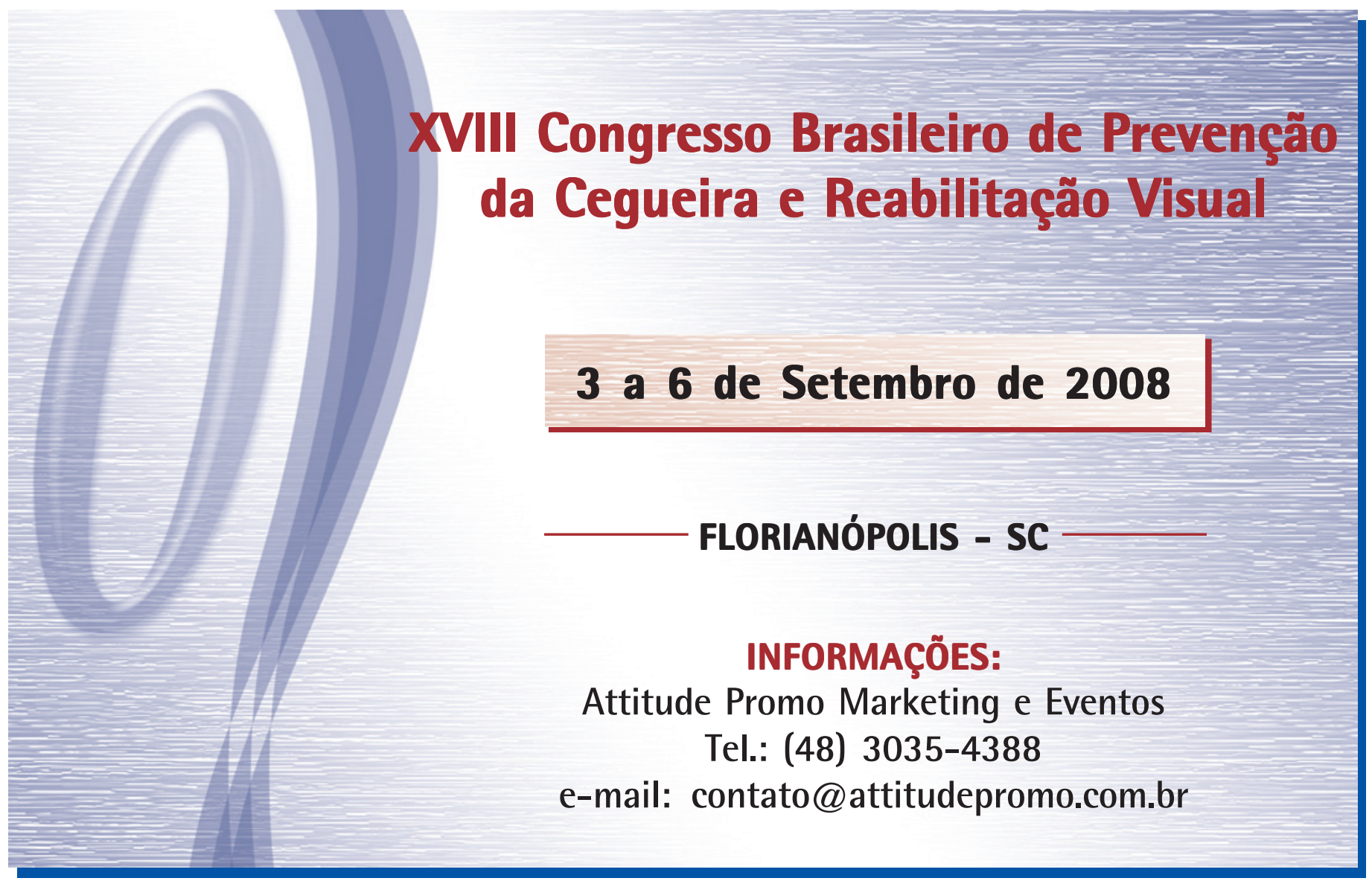

